\title{
Anti-PD-1 antibody significantly increases therapeutic efficacy of Listeria monocytogenes (Lm)-LLO immunotherapy
}

Mikayel Mkrtichyan', Namju Chong ${ }^{2}$, Rasha Abu Eid', Anu Wallecha ${ }^{3}$, Reshma Singh $^{3}$, John Rothman ${ }^{3}$ and Samir N Khleif ${ }^{*}$

\begin{abstract}
Background: One of the significant tumor immune escape mechanisms and substantial barrier for successful immunotherapy is tumor-mediated inhibition of immune response through cell-to-cell or receptor/ligand interactions. Programmed death receptor-1 (PD-1) interaction with its ligands, PD-L1 and PD-L2, is one of the important strategies that many tumors employ to escape immune surveillance. Upon PD-Ls binding to PD-1, T cell receptor (TCR) signaling is dampened, causing inhibition of proliferation, decreased cytokine production, anergy and/or apoptosis. Thus PD-Ls expression by tumor cells serves as a protective mechanism, leading to suppression of tumor-infiltrating lymphocytes in the tumor microenvironment. Lm-LLO immunotherapies have been shown to be therapeutically effective due to their ability to induce potent antigen-specific immune responses. However, it has been demonstrated that infection with $L m$ leads to up-regulation of PD-L1 on mouse immune cells that can inhibit effector T cells through PD-1/PD-L1 pathway.
\end{abstract}

Methods: Therapeutic and immune efficacy of Listeria-based vaccine (Lm-LLO-E7) in combination with anti-PD-1 antibody was tested in E7 antigen expressing TC-1 mouse tumor model. Tumor growth, survival, as well as peripheral and tumor-infiltrating immune cell profiles after immunotherapy were assessed.

Results: Here we demonstrate that the combination of an Lm-LLO immunotherapy with anti-PD-1 antibody that blocks PD-1/PD-L1 interaction, significantly improves immune and therapeutic efficacy of treatment in TC-1 mouse tumor model. Importantly, we show that in addition to significant reduction of regulatory T cells (Treg) and myeloid-derived suppressor cells (MDSC) in both spleen and tumor microenvironment that are mediated solely by the Lm-LLO immunotherapy, the addition of anti-PD-1 antibody to the treatment results in significant increase of antigen-specific immune responses in periphery and CD8 T cell infiltration into the tumor. As a result, this combinational treatment leads to significant inhibition of tumor growth and prolonged survival/complete regression of tumors in treated animals.

We also demonstrate that in vitro infection with Lm results in significant upregulation of surface PD-L1 expression on human monocyte-derived dendritic cells suggesting the translational capacity of this finding.

Conclusions: Our findings demonstrate that combination of Lm-LLO-based vaccine with blocking of PD-1/PD-L1 interaction is a feasible approach with clinical translation potential that can lead to overall enhancement of the efficacy of anti-tumor immunotherapy.

Keywords: PD-1, Immunotherapy, Listeria-based vaccine, Combinational immunotherapy

\footnotetext{
* Correspondence: SKHLEIF@gru.edu

${ }^{1}$ Cancer Center, Georgia Regents University, 1120 15th Street, Augusta

GA 30192, USA

Full list of author information is available at the end of the article
} 


\section{Background}

Listeria monocytogenes $(\mathrm{Lm})$ is a Gram-positive facultative anaerobic intracellular bacterium that has been extensively studied as a vaccine vector for several diseases. The unique intracellular life cycle of $\mathrm{Lm}$ in an antigenpresenting cell (APC) allows antigen to be processed and presented in the context of both MHC I and II molecules, resulting in strong $\mathrm{CD}^{+}$and $\mathrm{CD}^{+} \mathrm{T}$ cellmediated immune responses [1]. The listeriolysin-O (LLO) protein is the major virulence factor of $\mathrm{Lm}$ responsible for the lysis of the phagolysosome vacuole. Recently, LLO has been shown to be PAMP-like molecule by stimulating production of proinflammatory cytokines and inducing maturation of antigen-presenting cells [2]. Previously published reports have shown that genetically fusing an HPV16-E7 to a non-functional truncated form of LLO enhances the immunogenicity of antigens, as compared to the antigen expressed alone in the same system [3]. Furthermore, this enhanced immunogenicity correlates with a better therapeutic efficacy against established tumors $[3,4]$.

One of several mechanisms of tumor-mediated immune suppression is the expression of co-inhibitory molecules by tumor. Upon engagement to their ligands these molecules can suppress effector lymphocytes in the periphery and in the tumor microenvironment $[5,6]$. The PD-1 is one of the central signaling molecules that may inhibit $\mathrm{T}$ cell immunity when bound to its ligands (PD-L1 or PD-L2) by inducing T cell apoptosis and anergy [7]. PD-1 is expressed on the surface of activated lymphocytes and myeloid cells [8]. PD-L1 is expressed on activated T cells, $B$ cells, dendritic cells and macrophages, in addition to a wide range of non-hematopoietic cells [9]. PD-L1 is upregulated on numerous human tumors, and its expression has been shown to inversely correlate with survival in different types of cancer [10-15]. The expression of PD-L2 on various tumor cells was also demonstrated [16,17].

It has been shown that tumor eradication can be enhanced by PD-L1/PD-1 blockade [18-23]. Recently we demonstrated that the combination of PD-1 targeting with vaccine and low-dose cyclophosphamide significantly enhances antigen-specific immune responses, decreases tumor burden and increases survival of treated mice $[22,24]$.

Interestingly, in addition to significant immune and therapeutic potency of listeria-based immunotherapy [3,4], it has been demonstrated that infection with listeria lead to up-regulation of PD-L1 on immune cells [25].

Thus, we hypothesized that combination of Listeriabased vaccine with blockade of PD-1/PD-L interaction could improve the overall anti-tumor efficacy of immunotherapy.

Here we tested the therapeutic efficacy and immune mechanisms of anti-PD-1 antibody combined with listeria expressing LLO and E7 antigen (Lm-LLO-E7) in TC-1 tumor model.

\section{Methods}

Animals, cells lines, vaccine and other reagents

Six to eight weeks old female C57BL6 mice were purchased from NCI Frederick and kept under pathogen-free conditions. Mice were cared for under protocols approved by the NCI Animal Care and Use Committee. TC-1 cells that were derived by co-transfection of human papillomavirus strain 16 (HPV16) early proteins 6 and 7 (E6 and E7) and activated ras oncogene to primary $\mathrm{C} 57 \mathrm{BL} / 6$ mouse lung epithelial cells were obtained from ATCC (Manassas, VA), and cells were grown in RPMI 1640 supplemented with $10 \%$ FBS, penicillin and streptomycin $(100 \mathrm{U} / \mathrm{ml}$ each) and L-glutamine $(2 \mathrm{mM})$ at $37^{\circ} \mathrm{C}$ with $5 \% \mathrm{CO}_{2}$. Listeria vaccine vectors with or without human papilloma virus-16 (HPV-16) E7 (Lm-LLO and Lm-LLO-E7) provided by Advaxis Inc. were generated as described previously [3]. Both Lm-LLO and Lm-LLO-E7 were injected intraperitonealy (i.p.) at $5 \times 10^{6} \mathrm{CFU} /$ mouse dose. The antiPD-1 monoclonal antibody was obtained from CureTech (Israel) and was injected intravenously (i.v.) at a dose of $50 \mu \mathrm{g} /$ mouse. All fluorescently labeled antibodies and appropriate isotype controls used for flow cytometry were purchased from BD Biosciences (San Jose, CA) or eBiosciences (San Diego, CA).

\section{Mouse and human dendritic cell isolation, purification and analysis of PD-L1 expression}

Mouse dendritic cells (DC) were isolated and purified from bone marrow as we described earlier [26]. To obtain human DC, monocytes were isolated from healthy adult blood donors (National Institute of Health, Blood bank). Briefly, peripheral blood mononuclear cells (PBMC) were isolated from gradient centrifugation using Ficoll-Paque Plus (Amersham Biosciences) and, after washing, allowed to adhere to tissue culture plates for $2 \mathrm{~h}$ at $37^{\circ} \mathrm{C}$. Nonadherent cells were removed by washing, and the adherent monocytes were cultured in a plate at $37^{\circ} \mathrm{C}, 5 \% \mathrm{CO} 2$ in complete RPMI 1640 consisting of RPMI 1640, 2 mM Lglutamine, penicillin $(100 \mathrm{U} / \mathrm{ml})$, streptomycin $(100 \mathrm{ug} / \mathrm{ml})$, $10 \mathrm{mM}$ HEPES, 10\% fetal bovine serum, $10 \mathrm{mM}$ nonessential amino acids, $1 \mathrm{mM}$ sodium pyruvate, and $5 \times 10^{-5} \mathrm{M} 2$ mercaptoethanol. Cells were cultured in the presence of GM-CSF $(1000 \mathrm{U} / \mathrm{ml})$ and IL-4 $(500 \mathrm{U} / \mathrm{ml})$ for 4 days to become immature DCs. GM-CSF and IL-4 were added again along with fresh medium on day 3 . The DC viability in cultures was assessed using the trypan blue exclusion protocol. Trypan blue-negative cells were considered alive. After culturing DCs from monocytes for 4-5 days, DCs were collected and transferred to 6 well plate $\left(1 \times 10^{6}\right.$ cells $\left./ \mathrm{ml}\right)$. Different concentrations of Lm-LLO or Lm-LLO-E7 were added to DCs culture (0, 
$10^{7}, 10^{8}$, and $10^{9} \mathrm{CFU} / \mathrm{ml}$ ) for an hour followed by adding gentamicin $(50 \mathrm{ug} / \mathrm{ml})$ to kill listeria, and cultured for $48 \mathrm{hr}$.

Both mouse and human DCs were stained with appropriate fluorescently labeled anti-PD-L1 antibody (PE anti-mouse PD-L1 and FITC anti-human PD-L1). Isotypematched mAbs were used as negative controls. The stained cells were analyzed using FACSCalibur cytometer and CellQuest software (BD Biosciences).

\section{Tumor implantation and treatment}

The therapeutic experiments aimed to analyze tumor growth and survival were performed as described earlier $[22,24]$. Briefly, mice were implanted with 50,000 TC-1 cells/mouse subcutaneous (s.c.) in the right flank on day 0 . On day 8 (tumor size $\sim 3-4 \mathrm{~mm}$ in diameter), animals from appropriate groups (5 mice per group) were injected i.p. with Lm-LLO or Lm-LLO-E7 with or without anti-PD-1 $\mathrm{Ab}$ i.v. Mice were treated with vaccine and anti-PD-1 Ab one more time on day 15 after tumor implantation. Another group of mice remained non-treated. Tumors were measured every 3-4 days using digital calipers, and tumor volume was calculated using the formula $\mathrm{V}=\left(\mathrm{W}^{2} \times \mathrm{L}\right) / 2$, whereby $\mathrm{V}$ is volume, $\mathrm{L}$ is length (longer diameter) and $\mathrm{W}$ is width (shorter diameter). In these experiments mice were sacrificed when mice became moribund, tumors were ulcerated or tumor volume reached $1.5 \mathrm{~cm}^{3}$.

In immunologic experiments same groups of mice were treated similarly, except mice were sacrificed six days after the second treatment, on day 21. Spleens and tumors were isolated and analyzed for antigen-specific immune responses, CD8 T cells, Tregs and myeloid derived suppressor cells (MDSC).

\section{Analysis of antigen-specific cellular immune responses, Tregs, MDSC in periphery and tumors}

ELISPOT was used to detect IFNY production in E7restimulated $(10 \mu \mathrm{g} / \mathrm{ml})$ splenocyte cultures from treated and control mice, as suggested by the manufacturer (BD Biosciences, San Jose, CA). A CTL Immunospot Analyzer (Cellular Technology Ltd., Shaker Heights, OH) was used to analyze spots. The number of spots from irrelevant peptide (hgp 100 $25-33^{-}$KVPRNQDWL-Celtek Bioscience, Nashville, TN) re-stimulated splenocytes were subtracted from E7-restimulated cultures.

Tumor samples were processed using GentleMACS Dissociator and the solid tumor homogenization protocol, as suggested by the manufacturer (Miltenyi Biotec, Auburn, $\mathrm{CA})$. The number of tumor-infiltrating $\mathrm{CD}^{+}, \mathrm{CD}^{+} \mathrm{Foxp}^{+}$ (Treg) and $\mathrm{CD} 11 \mathrm{~b}^{+} \mathrm{Gr}-\mathrm{1}^{+}$(MDSC) cells were analyzed within $\mathrm{CD} 45^{+}$hematopoietic cell population using flow cytometry assay as we described earlier $[22,26]$. The level of Treg cells and MDSC was also evaluated in spleens of tumor-bearing treated and control mice using the same flow cytometry assay.

\section{Statistical analysis}

All statistical parameters (average values, SD for PD-L1 expression on DC, tumor volumes, ELISPOT and peripheral and tumor-infiltrating cell analysis) and statistical significance between groups (for peripheral and tumorinfiltrating cell analysis) were calculated using GraphPad Prism Software (San Diego, CA). Statistical significance between groups was determined by one-way ANOVA with Tukey's multiple comparison post-test $(P<0.05$ was considered statistically significant).

\section{Results}

Infection of murine DC with Lm-LLO and Lm-LLO-E7 upregulates surface PD-L1 expression

It was previously demonstrated that mouse splenocytes infection with $\mathrm{Lm}$ results in significant upregulation of PDL1 expression on the majority of cells, and that the level of PD-L1 expression was highest among CD11 ${ }^{+}$DC [25]. Considering the importance of $\mathrm{DC}$ in priming immune response and inhibitory role of PD-1/PD-L1 interaction, we decided first to analyze the effect of Lm-LLO and LmLLO-E7 on the PD-L1 expression on DC. To avoid the influence of cell-cell interactions within the mixed cell population on the accuracy of results, we tested the effect of different concentrations of Lm-LLO and Lm-LLO-E7 on PD-L1 expression on the surface of purified CD11c ${ }^{+}$ DCs. As shown in Figure 1, both Lm-LLO and LmLLO-E7 significantly upregulate PD-L1 expression at $10^{8}$ and $10^{9} \mathrm{CFU} / \mathrm{ml}$ doses in a dose dependent manner. Importantly, there were no differences detected between Lm-LLO- and Lm-LLO-E7-induced PD-L1 upregulation on DC at any of the tested doses (Figure 1), indicating that this effect is antigen-independent.

\section{Anti-PD-1 enhances therapeutic efficacy of Lm-LLO-E7 vaccine}

After confirming the effect of Lm-LLO and Lm-LLO-E7 on upregulation of PD-L1 expression on DC, and considering the inhibitory effect of PD-1/PD-L1 interaction we hypothesize that combination of PD-1/PD-L1 blockade with Listeria-based vaccine could improve the anti-tumor efficacy of immunotherapy. To test this hypothesis we evaluated the effect of anti-PD-1 Ab and Lm-LLO-E7 combination on tumor growth and survival of mice in TC-1 tumor model based on E7-expressing lung epithelial cells. We deliberately used a low dose of Lm-LLO-E7, delayed treatment schedule and implanted a high number of tumor cells in order to minimize the effect of vaccine alone. Mice were implanted with 50,000 TC-1 cells s.c. on day 0 , and on days 8 and 15 after tumor implantation mice 


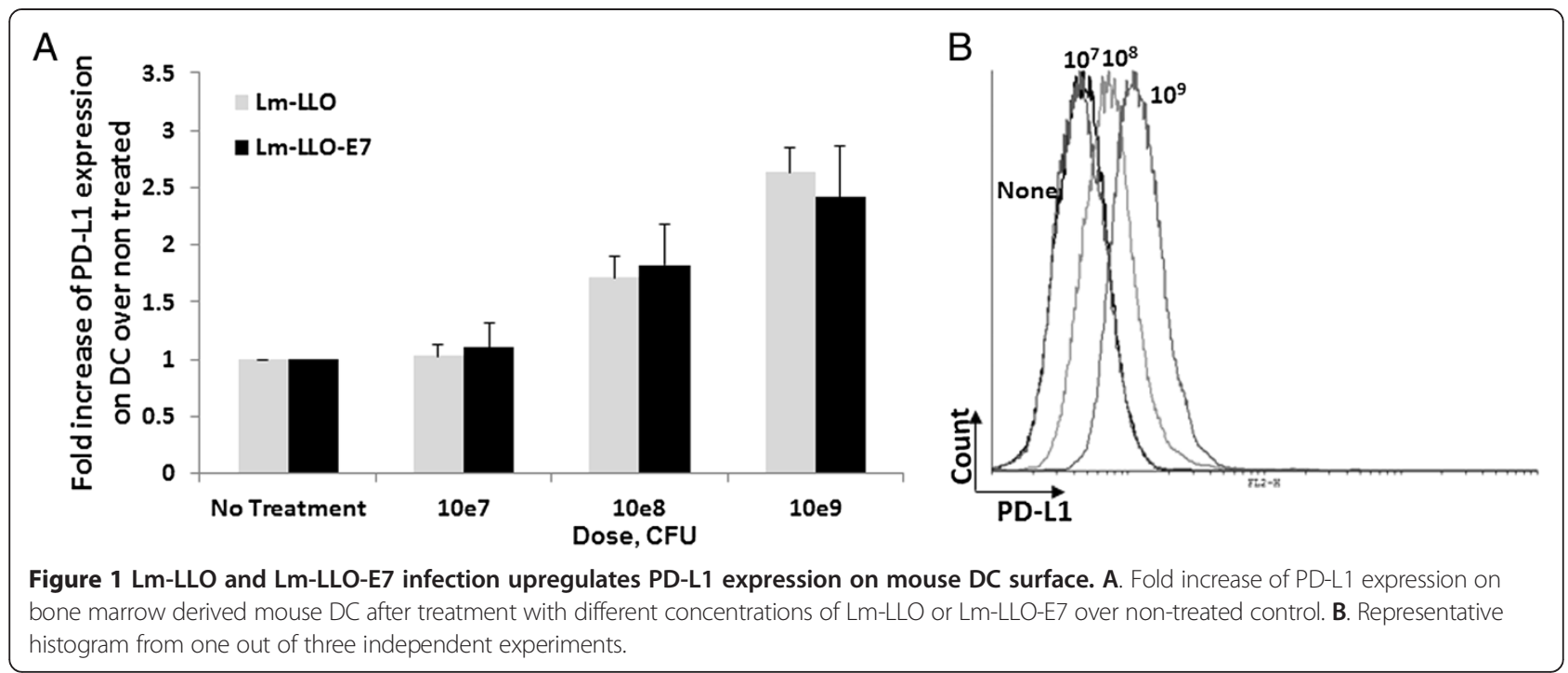

were injected with Lm-LLO-E7 or Lm-LLO with or without anti-PD-1 Ab (Figure 2A). Another group of mice remained non-treated.

While Lm-LLO-E7 vaccine alone resulted in slight inhibition of tumor growth, Lm-LLO-E7/anti-PD-1 combination significantly slowed tumor growth (Figure 2B) and resulted in prolonged survival and complete tumor regression in $20 \%$ of treated mice (Figure $2 \mathrm{C}$ ).

These experiments reveal that combination of antiPD-1 Ab with Lm-LLO-E7 vaccine is a feasible strategy

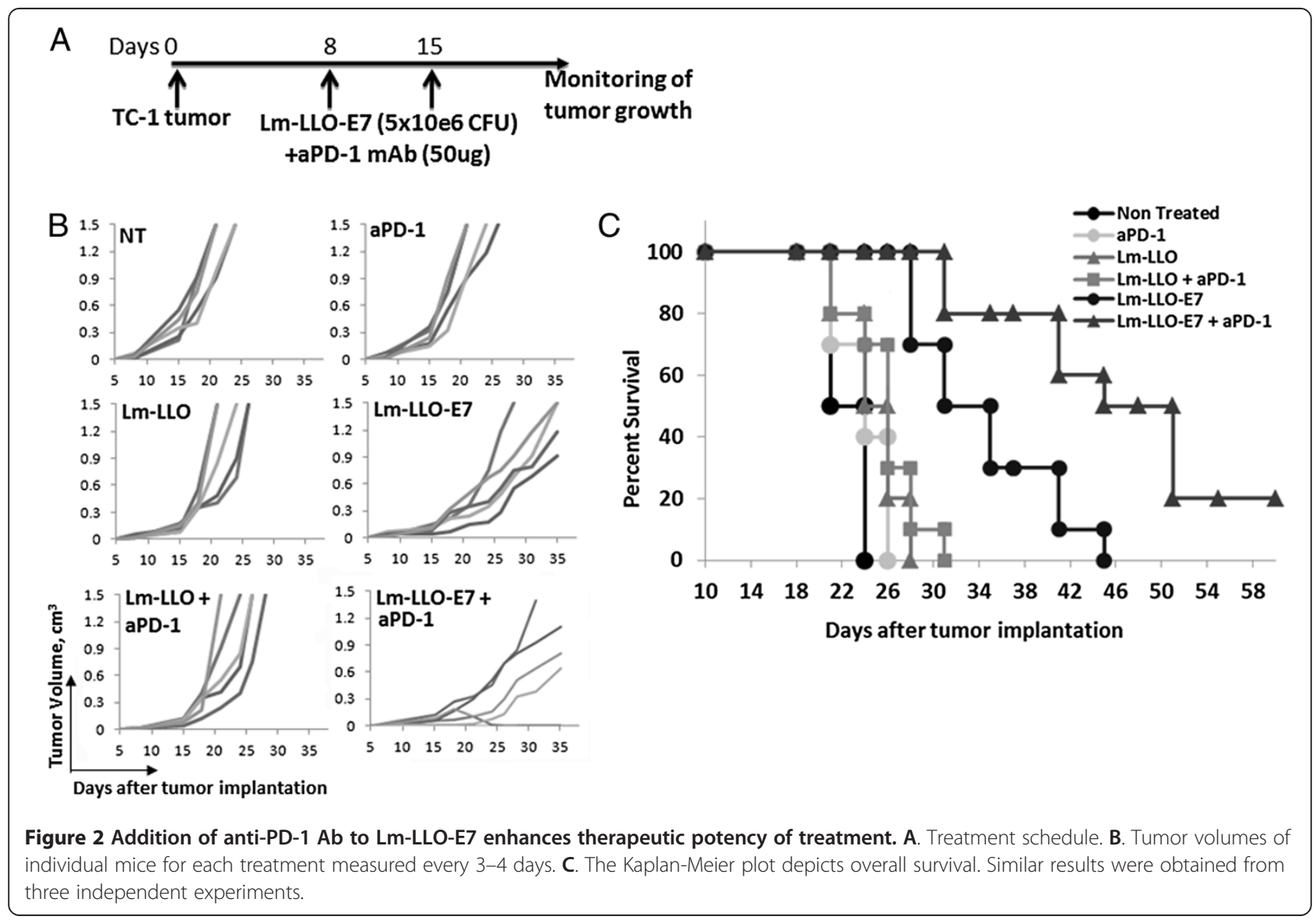


resulting in tumor growth inhibition and improved survival even at stringent conditions that were used in these experiments.

Combination of anti-PD-1 Ab and Lm-LLO-E7 significantly enhances antigen-specific immune responses and CD8 T cell infiltration into the tumor

To define the immune mechanism and evaluate the immunologic efficacy of Lm-LLO-E7/anti-PD-1 Ab combination we next assessed the levels of antigen-specific IFN $\gamma$-producing cells in spleens from treated tumorbearing mice and tumor-infiltrated CD8 T cells. Mice were implanted with TC-1 cells and treated as described above for therapeutic experiments, except, six days after the second treatment mice were sacrificed and spleens and tumors were harvested. Analysis of E7-specific IFN $\gamma$ producing cells was performed using a standard ELISPOT assay. As expected, treatment with Lm-LLO-E7 alone induced significant levels of IFNy-producing E7-specific cells compared to controls $(\mathrm{P}<0.001)$. Notably, addition of $\mathrm{PD}-1$ /PD-L1 blockade with anti-PD-1 Ab, to Lm-LLO-E7 resulted in further significant increase in antigen specific immune response when compared to Lm-LLO-E7 alone $(\mathrm{P}<0.01)$ (Figure 3A).

To further determine the mechanism by which combining Lm-LLO-E7/anti-PD-1 Ab exerts its therapeutic effect, we tested the influence of treatment on tumorinfiltrated CD8 T cells. Tumor-infiltrated CD8 T cells were tested on day 21 post tumor implantation in mice treated as described above. As expected, Lm-LLO-E7 and Lm-LLO-E7/anti-PD-1 Ab showed a significant increase in tumor-infiltrated CD8 $\mathrm{T}$ cells compared to control groups $(\mathrm{P}<0.05$ for Lm-LLO-E7 alone and $\mathrm{P}<$ 0.001 for Lm-LLO-E7/anti-PD-1 Ab) (Figure 3B). Similar to peripheral immune response, addition of anti-PD-1 Ab to Lm-LLO-E7 treatment resulted in significant increase in CD8 T cell tumor infiltration compared to Lm-LLO-E7 alone $(\mathrm{P}<0.05)$ (Figure $3 \mathrm{~B})$.

Lm-LLO treatment significantly reduces both splenic and tumor-infiltrated MDSC and Treg cells regardless of presence of antigen or anti-PD-1 Ab

Two cell subsets with profound immune response inhibitory activity are MDSC and Treg cells. Accordingly, we analyzed these subsets both in periphery and within tumor microenvironment to understand the impact of Lm-LLO-E7/anti-PD-1 Ab combinational treatment. Spleens and tumors harvested six days after second vaccination were assessed for percent (spleen) and actual numbers (tumors) of MDSC and Treg cells. While the percent of MDSC in spleens of tumor-free animals is about $2.5 \%$, in presence of tumor this percent significantly increases $(\sim 15 \%)$ (Figure 4A). Interestingly, treatment with Lm-LLO, regardless of presence of E7 antigen or anti-PD-1 treatment, significantly decreases the levels of MDSC in spleens compared to control animals $(\mathrm{P}<0.05)$ (Figure $4 \mathrm{~A})$. Similarly, numbers of tumor-infiltrated MDSC also were significantly decreased after treatment with Lm-LLO, Lm-LLO-E7 and Lm-LLO-E7/anti-PD-1 Ab treatment (Figure 4B). Importantly, Treg cells in both spleens (Figure 5A) and tumors (Figure 5B) were also slightly but significantly

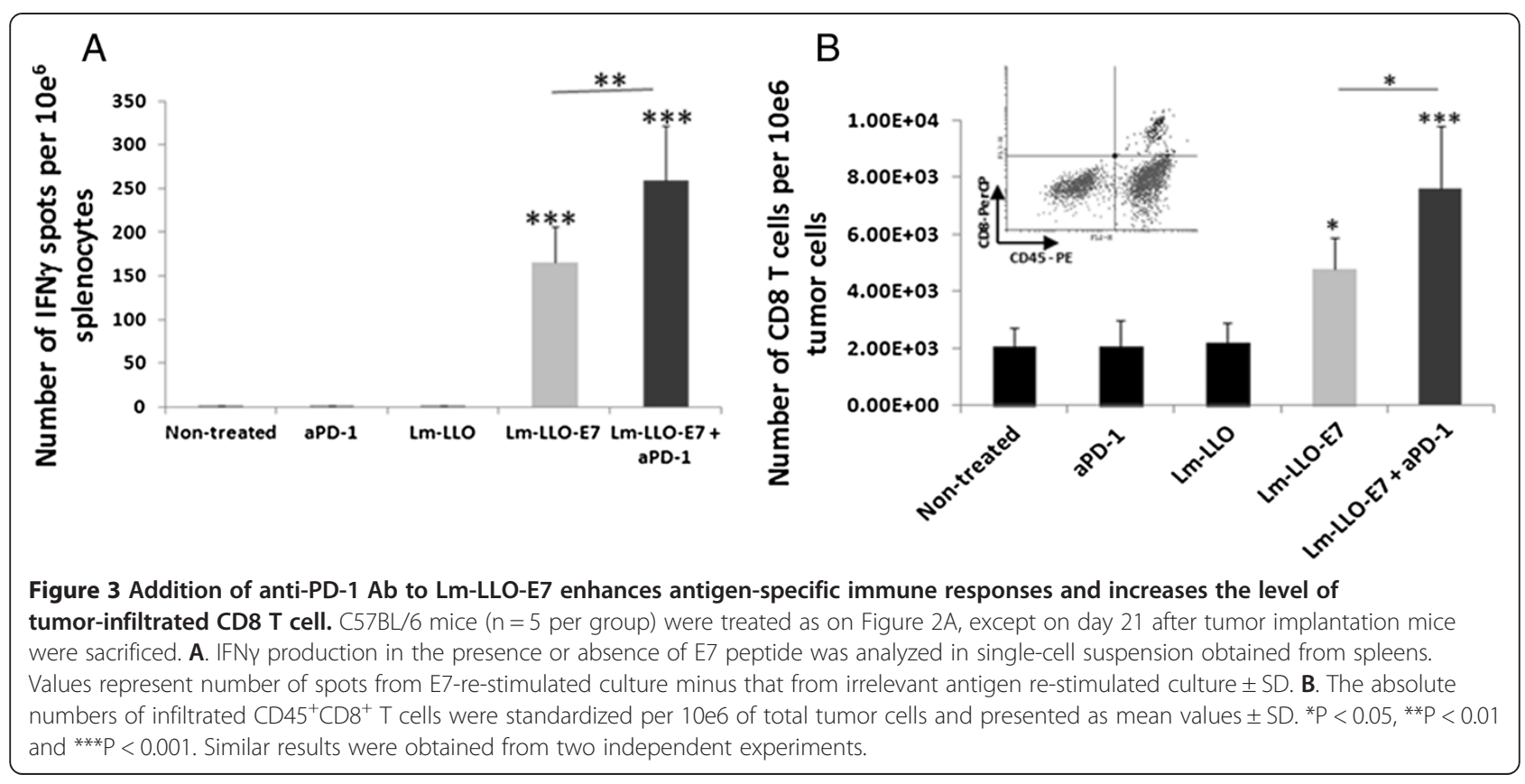




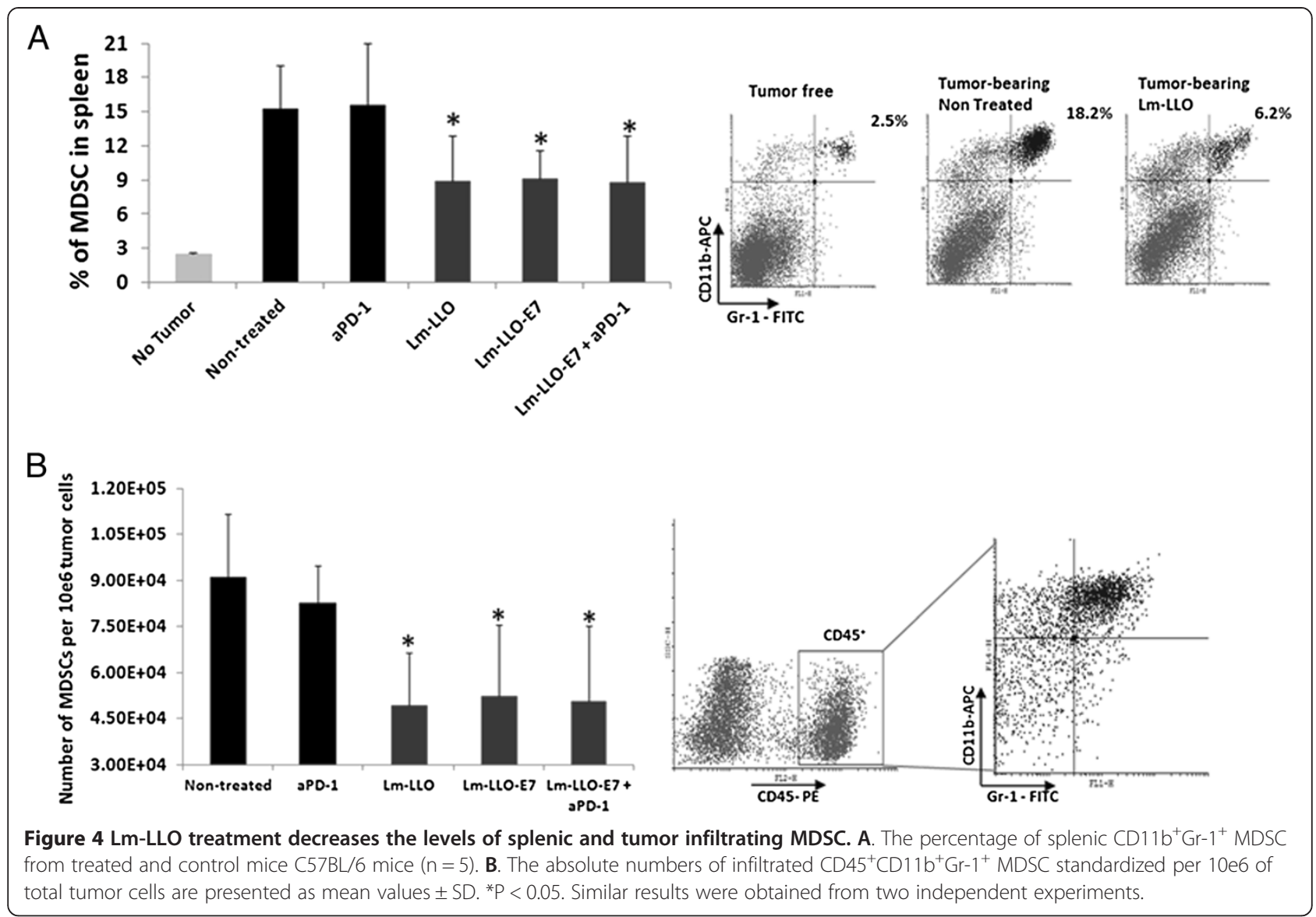

decreased in groups treated with Lm-LLO either alone or with E7 or anti-PD-1 Ab.

These data suggest that Lm-LLO is solely responsible for decrease of MDSC and Tregs in both spleens and tumors of treated mice, and that the addition of antigen or anti-PD-1 antibody does not affect levels of these cells.

\section{Infection of human DC with Lm-LLO also leads to upreguation of surface PD-L1 expression}

After demonstrating the therapeutic efficacy and immune mechanism by which Lm-LLO-E7/anti-PD-1 Ab combination exerts anti-tumor effect, we decided to test if LmLLO also affect the levels of PD-L1 expression on human DC and so as, to understand if our findings could be translated into the clinic. Monocyte-derived human DC were isolated from PBMC of healthy volunteers as described in Methods section. Human DC were infected with different concentrations of Lm-LLO and Lm-LLO -E7. We found that, similar to murine DC, both Lm-LLO and Lm-LLO-E7 infection leads to significant upregulation of surface PD-L1 (Figure 6A and B and data not shown). As for murine DC, the PD-L1 upregulation on human DC was dose dependent. This finding suggests that combination of listeria-based vaccine with anti-PD-1 $\mathrm{Ab}$ could be a potent and clinically translatable immunotherapeutic approach.

\section{Discussion}

The PD-1/PD-L1 engagement has been shown to dampen TCR signaling, decrease cytokine production by the $\mathrm{T}$ cell, thus, reducing proliferation, anergy and/or apoptosis [27,28]. Accordingly, PD-L1 expression by tumor cells serves a protective function that can lead to suppression of tumor-infiltrating effector lymphocytes thus, allowing tumor to escape immune surveillance [29-31]. On the other hand, in disease-free conditions, PD-1/PD-L1 interaction serves as an important factor in preventing autoimmunity $[9,32]$.

It was previously demonstrated that Listeria-based tumor antigen delivery is a therapeutically potent strategy capable of inducing robust antigen-specific immune responses, inhibiting tumor growth and prolonging survival in pre-clinical mouse tumor models $[3,4]$ and is currently in clinical trials. A Phase 1 study has been completed with Lm-LLO-E7 [33] and 4 Phase 2 clinical trials are active or about to be initiated [34].

Interestingly, despite the promising therapeutic potency of Listeria-based immunotherapies, it was shown that 

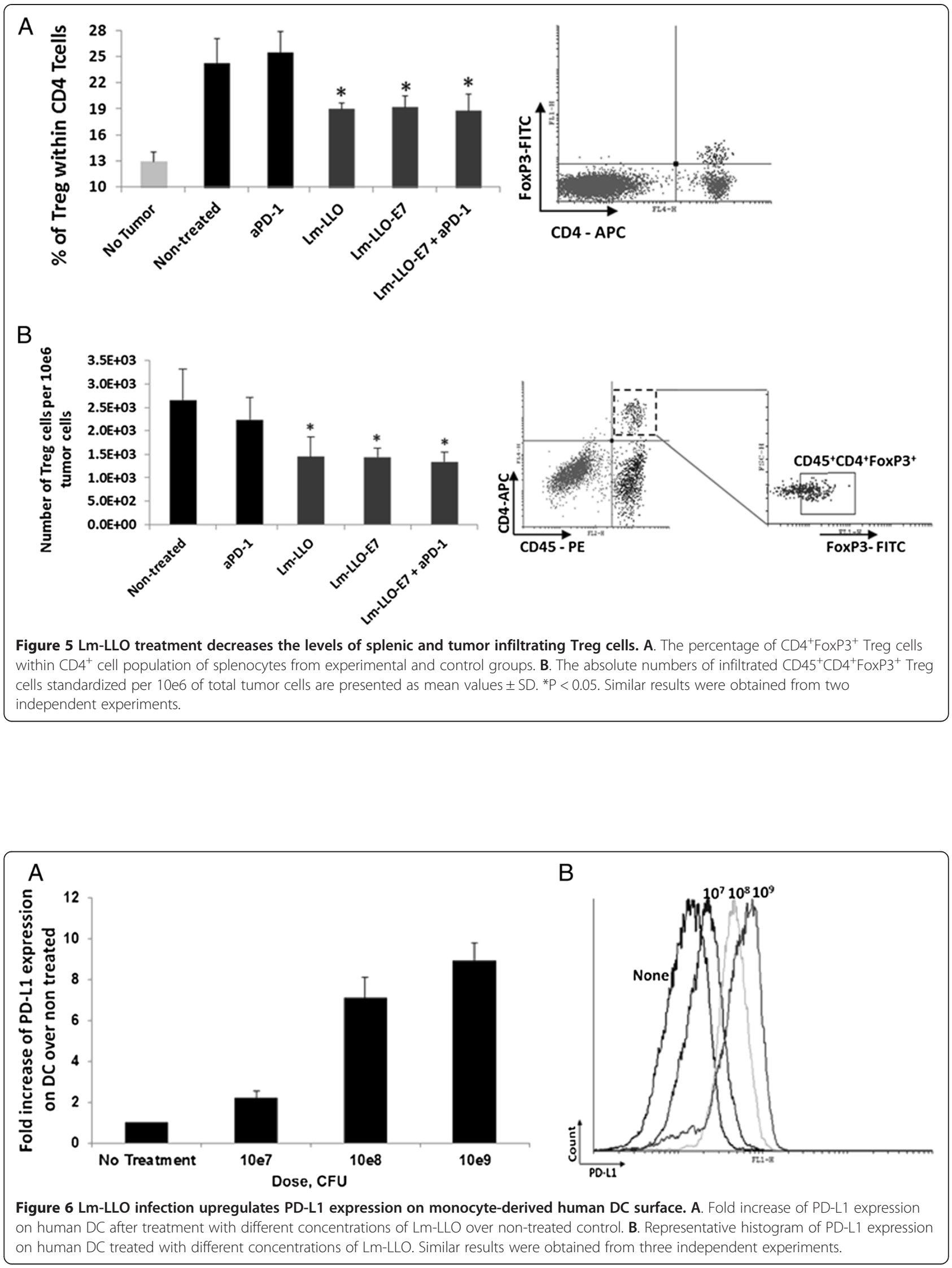
infection with listeria leads to up-regulation of PD-L1 on immune cells [25]. This observation allowed us to hypothesize, that combination of PD-1/PD-L1 blockade with Listeria-based immunotherapy will result in even more potent anti-tumor efficacy of treatment. To test this hypothesis we first confirmed the effect of Listeria infection on PD-L1 expression on both mouse and human DCs and then evaluated the immune and therapeutic efficacy of Lm-LLO-E7 combination with anti-PD-1 Ab in mouse TC-1 tumor model. To minimize the effect of Listeria vaccine alone and thus, understand the impact of PD-1/PDL1 interaction blockade, we used delayed treatment schedule and low dose of Lm-LLO-E7. We demonstrate that even at these stringent conditions addition of anti-PD- $1 \mathrm{Ab}$ to Listeria-based treatment results in improvement of tumor-growth inhibition and prolongation of survival and significantly stronger antigen-specific immune response compared to Listeria vaccine alone.

We showed previously that combination of anti-PD-1 Ab with peptide vaccine requires incorporation of single low dose of cyclophosphamide (CPM) treatment to decrease Treg cell numbers, and that potent therapeutic efficacy is seen only when all three components are combined [22,24]. Interestingly, here we found that while addition of anti-PD $-1 \mathrm{Ab}$ to Lm-LLO-E7 also significantly increases infiltration of CD8 T cells into the tumor, it does not affect the levels of neither peripheral nor tumor-infiltrated suppressor cells. Instead, surprisingly, Lm-LLO regardless of the presence of antigen or PD-1/PD-L1 interaction blockade, results in significant decrease of Treg cells and MDSC in both spleens and tumors of treated animals. While we previously demonstrated that Lm-LLO is able to decrease tumor-infiltrated Treg cells [34], here we demonstrate for the first time, to our knowledge, that Lm-LLO treatment results in decrease of MDSC. Although the mechanisms for both Lm-LLO-induced Treg cells and MDSC decrease are still unknown and currently under investigation in our laboratories, here we demonstrate that these events along with PD-1/PD-L1 interaction blockade with anti-PD-1 Ab lead to increased antigen-specific immune responses and thus, result in enhancement of overall anti-tumor efficacy of treatment.

\section{Conclusions}

Overall, our findings demonstrate that combination of $L m$-LLO-based vaccine with anti-PD-1 Ab leads to increased antigen-specific immune responses and tumor-infiltrating CD8 T cell, decrease in suppressor cells (Treg cells and MDSC) and as a result, leads to significant inhibition of tumor growth and prolonged survival/complete regression of tumors in treated animals. Thus, we show that combination of $L m$-LLO-based vaccine with blocking of PD-1/PD-L1 interaction is a feasible and translatable approach that can lead to overall enhancement of the efficacy of anti-tumor immunotherapy.

\section{Abbreviations}

PD-1: Programmed death receptor-1; PD-L1/2: Programmed death receptor-1 ligand1/2; TCR: T cell receptor; Lm: Listeria monocytogene; LLO: ListeriolysinO; Treg: Regulatory T cell, MDSC- myeloid-derived suppressor cells; PAMP: Pathogen-associated molecular pattern; HPV16: Human papillomavirus strain 16; CFU: Colony-forming unit; DC: Dendritic cell; PBMC: Peripheral blood mononuclear cells; PE: Phycoerythrin; FITC: Fluorescein isothiocyanate; Ab: Antibody; CTL: Cytotoxic T lymphocyte; CPM: Cyclophosphamide.

\section{Competing interests}

AW, RS and JR are current or former employees of Advaxis Inc. which provided Lm-LLO and Lm-LLO-E7. RS and JR are shareholders of Advaxis Inc. Other authors declare no conflict of interests.

\section{Authors' contributions}

MM and SK were the main investigators and take primary responsibility for the paper. MM performed the in vivo experiments and immune response analysis, RAE performed tumor infiltration analysis, NC carried out the in vitro experiments with dendritic cells. AW, RS and JR participated in the design and coordination, and helped to draft the manuscript. MM and SK wrote the paper. All authors read and approved the final manuscript.

\section{Acknowledgment}

This work was supported by the Intramural Research Program of the Center for Cancer Research, NCl, NIH, Georgia Regents University Cancer Center (GRUCC) and Advaxis Inc. RAE is supported by a Fellowship Grant from King Hussein Institute for Biotechnology and Cancer (KHIBC, Jordan).

\section{Author details}

'Cancer Center, Georgia Regents University, 1120 15th Street, Augusta GA 30192, USA. ${ }^{2}$ National Cancer Institute, NIH, Vaccine Branch, Bethesda, MD 20892, USA. ${ }^{3}$ Advaxis Inc., Princeton, NJ 08540, USA.

Received: 28 June 2013 Accepted: 20 August 2013

Published: 29 August 2013

\section{References}

1. Pamer EG: Immune responses to Listeria monocytogenes. Nat Rev Immunol 2004, 4:812-823.

2. Wallecha A, Wood L, Pan ZK, Maciag PC, Shahabi V, Paterson Y: Listeria monocytogenes-derived listeriolysin $\mathrm{O}$ has pathogen-associated molecular pattern-like properties independent of its hemolytic ability. Clin Vaccine Immunol 2013, 20:77-84.

3. Gunn GR, Zubair A, Peters C, Pan ZK, Wu TC, Paterson Y: Two Listeria monocytogenes vaccine vectors that express different molecular forms of human papilloma virus-16 (HPV-16) E7 induce qualitatively different T cell immunity that correlates with their ability to induce regression of established tumors immortalized by HPV-16. I Immunol 2001, 167:6471-6479.

4. Sewell DA, Shahabi V, Gunn GR 3rd, Pan ZK, Dominiecki ME, Paterson Y: Recombinant Listeria vaccines containing PEST sequences are potent immune adjuvants for the tumor-associated antigen human papillomavirus-16 E7. Cancer Res 2004, 64:8821-8825.

5. Hamanishi J, Mandai M, Iwasaki M, Okazaki T, Tanaka Y, Yamaguchi K, Higuchi T, Yagi H, Takakura K, Minato N, et al: Programmed cell death 1 ligand 1 and tumor-infiltrating CD8+ T lymphocytes are prognostic factors of human ovarian cancer. Proc Natl Acad Sci USA 2007, 104:3360-3365.

6. Tsushima F, Tanaka K, Otsuki N, Youngnak P, Iwai H, Omura K, Azuma M: Predominant expression of $\mathrm{B} 7-\mathrm{H} 1$ and its immunoregulatory roles in oral squamous cell carcinoma. Oral Oncol 2006, 42:268-274.

7. Francisco LM, Sage PT, Sharpe AH: The PD-1 pathway in tolerance and autoimmunity. Immunol Rev 2010, 236:219-242.

8. Agata Y, Kawasaki A, Nishimura H, Ishida Y, Tsubata T, Yagita H, Honjo T: Expression of the PD-1 antigen on the surface of stimulated mouse $T$ and B lymphocytes. Int Immunol 1996, 8:765-772.

9. Keir ME, Liang SC, Guleria I, Latchman YE, Qipo A, Albacker LA, Koulmanda M, Freeman GJ, Sayegh MH, Sharpe AH: Tissue expression of PD-L1 mediates peripheral T cell tolerance. J Exp Med 2006, 203:883-895.

10. Ohigashi Y, Sho M, Yamada Y, Tsurui Y, Hamada K, Ikeda N, Mizuno T, Yoriki R, Kashizuka H, Yane K, et al: Clinical significance of programmed death-1 
ligand-1 and programmed death-1 ligand-2 expression in human esophageal cancer. Clin Cancer Res 2005, 11:2947-2953.

11. Konishi J, Yamazaki K, Azuma M, Kinoshita I, Dosaka-Akita H, Nishimura M: B7-H1 expression on non-small cell lung cancer cells and its relationship with tumor-infiltrating lymphocytes and their PD-1 expression. Clin Cancer Res 2004, 10:5094-5100.

12. Nomi T, Sho M, Akahori T, Hamada K, Kubo A, Kanehiro H, Nakamura S, Enomoto K, Yagita H, Azuma M, Nakajima Y: Clinical significance and therapeutic potential of the programmed death-1 ligand/programmed death-1 pathway in human pancreatic cancer. Clin Cancer Res 2007, 13:2151-2157.

13. Thompson RH, Gillett MD, Cheville JC, Lohse CM, Dong H, Webster WS, Krejci KG, Lobo JR, Sengupta S, Chen L, et al: Costimulatory B7-H1 in renal cell carcinoma patients: indicator of tumor aggressiveness and potential therapeutic target. Proc Natl Acad Sci U S A 2004, 101:17174-17179.

14. Ghebeh H, Mohammed S, Al-Omair A, Qattan A, Lehe C, Al-Qudaihi G, Elkum N, Alshabanah M, Bin Amer S, Tulbah A, et al: The B7-H1 (PD-L1) T lymphocyte-inhibitory molecule is expressed in breast cancer patients with infiltrating ductal carcinoma: correlation with important high-risk prognostic factors. Neoplasia 2006, 8:190-198.

15. Thompson RH, Kuntz SM, Leibovich BC, Dong H, Lohse CM, Webster WS, Sengupta S, Frank I, Parker AS, Zincke H, et al: Tumor B7-H1 is associated with poor prognosis in renal cell carcinoma patients with long-term follow-up. Cancer Res 2006, 66:3381-3385.

16. Messal N, Serriari NE, Pastor S, Nunes JA, Olive D: PD-L2 is expressed on activated human T cells and regulates their function. Mol Immunol 2011, 48:2214-2219.

17. Okazaki T, Honjo T: PD-1 and PD-1 ligands: from discovery to clinical application. Int Immunol 2007, 19:813-824.

18. Curiel TJ, Wei S, Dong H, Alvarez X, Cheng P, Mottram P, Krzysiek R, Knutson $\mathrm{KL}$, Daniel B, Zimmermann MC, et al: Blockade of B7-H1 improves myeloid dendritic cell-mediated antitumor immunity. Nat Med 2003, 9:562-567.

19. Strome SE, Dong H, Tamura H, Voss SG, Flies DB, Tamada K, Salomao D, Cheville J, Hirano F, Lin W, et al: B7-H1 blockade augments adoptive T-cell immunotherapy for squamous cell carcinoma. Cancer Res 2003, 63:6501-6505.

20. He YF, Zhang GM, Wang XH, Zhang H, Yuan Y, Li D, Feng ZH: Blocking programmed death-1 ligand-PD-1 interactions by local gene therapy results in enhancement of antitumor effect of secondary lymphoid tissue chemokine. J Immunol 2004, 173:4919-4928.

21. Blank C, Brown I, Peterson AC, Spiotto M, Iwai Y, Honjo T, Gajewski TF: $\mathrm{PD}-\mathrm{L} 1 / \mathrm{B} 7 \mathrm{H}-1$ inhibits the effector phase of tumor rejection by $\mathrm{T}$ cell receptor (TCR) transgenic CD8+ T cells. Cancer Res 2004, 64:1140-1145.

22. Mkrtichyan M, Najjar YG, Raulfs EC, Abdalla MY, Samara R, Rotem-Yehudar R, Cook L, Khleif SN: Anti-PD-1 synergizes with cyclophosphamide to induce potent anti-tumor vaccine effects through novel mechanisms. Eur J Immunol 2011, 41:2977-2986

23. Curran MA, Montalvo W, Yagita H, Allison JP: PD-1 and CTLA-4 combination blockade expands infiltrating $T$ cells and reduces regulatory T and myeloid cells within B16 melanoma tumors. Proc Natl Acad Sci U S A 2010, 107:4275-4280.

24. Mkrtichyan M, Najjar YG, Raulfs EC, Liu L, Langerman S, Guittard G, Ozbun L, Khleif SN: B7-DC-Ig enhances vaccine effect by a novel mechanism dependent on PD-1 expression level on T cell subsets. J Immunol 2012, 189:2338-2347.

25. Rowe JH, Johanns TM, Ertelt JM, Way SS: PDL-1 blockade impedes T cell expansion and protective immunity primed by attenuated Listeria monocytogenes. J Immunol 2008, 180:7553-7557.

26. Mkrtichyan M, Ghochikyan A, Davtyan H, Movsesyan N, Loukinov D, Lobanenkov V, Cribbs DH, Laust AK, Nelson EL, Agadjanyan MG: Cancertestis antigen, BORIS based vaccine delivered by dendritic cells is extremely effective against a very aggressive and highly metastatic mouse mammary carcinoma. Cell Immunol 2011, 270:188-197.

27. Keir ME, Latchman YE, Freeman GJ, Sharpe AH: Programmed death-1 (PD-1):PD-ligand 1 interactions inhibit TCR-mediated positive selection of thymocytes. J Immunol 2005, 175:7372-7379.

28. Blank C, Mackensen A: Contribution of the PD-L1/PD-1 pathway to T-cell exhaustion: an update on implications for chronic infections and tumor evasion. Cancer Immunol Immunother 2007, 56:739-745.

29. Freeman GJ, Long AJ, Iwai Y, Bourque K, Chernova T, Nishimura H, Fitz ${ }_{\text {, }}$ Malenkovich N, Okazaki T, Byrne MC, et al: Engagement of the PD-1 immunoinhibitory receptor by a novel B7 family member leads to negative regulation of lymphocyte activation. J Exp Med 2000, 192:1027-1034.

30. Parry RV, Chemnitz JM, Frauwirth KA, Lanfranco AR, Braunstein I, Kobayashi SV, Linsley PS, Thompson CB, Riley JL: CTLA-4 and PD-1 receptors inhibit T-cell activation by distinct mechanisms. Mol Cell Biol 2005, 25:9543-9553.

31. Seo SK, Seo HM, Jeong HY, Choi IW, Park YM, Yagita H, Chen L, Choi IH: Co-inhibitory role of T-cell-associated B7-H1 and B7-DC in the T-cell immune response. Immunol Lett 2006, 102:222-228.

32. Fife BT, Guleria I, Gubbels Bupp M, Eagar TN, Tang Q, Bour-Jordan H, Yagita $H$, Azuma M, Sayegh MH, Bluestone JA: Insulin-induced remission in newonset NOD mice is maintained by the PD-1-PD-L1 pathway. J Exp Med 2006, 203:2737-2747.

33. Maciag PC, Radulovic S, Rothman J: The first clinical use of a liveattenuated Listeria monocytogenes vaccine: a phase I safety study of Lm-LLO-E7 in patients with advanced carcinoma of the cervix. Vaccine 2009, 27:3975-3983.

34. Wallecha A, French C, Petit R, Singh R, Amin A, Rothman J: Lm-LLO-based immunotherapies and HPV-associated disease. J Oncol 2012, 2012:542851.

doi:10.1186/2051-1426-1-15

Cite this article as: Mkrtichyan et al:: Anti-PD-1 antibody significantly increases therapeutic efficacy of Listeria monocytogenes (Lm)-LLO immunotherapy. Journal for ImmunoTherapy of Cancer 2013 1:15.

\section{Submit your next manuscript to BioMed Central and take full advantage of:}

- Convenient online submission

- Thorough peer review

- No space constraints or color figure charges

- Immediate publication on acceptance

- Inclusion in PubMed, CAS, Scopus and Google Scholar

- Research which is freely available for redistribution 